Research Article

\title{
Prevalence of Urinary Tract Infection and Its Associated Factors among Pregnant Women in Ethiopia: A Systematic Review and Meta-Analysis
}

\author{
Temesgen Getaneh $\mathbb{D}^{1},{ }^{1}$ Ayenew Negesse, ${ }^{2,3}$ Getenet Dessie, ${ }^{4}$ Melaku Desta, ${ }^{1}$ \\ and Agimasie Tigabu ${ }^{5}{ }^{5}$ \\ ${ }^{1}$ Department of Midwifery, College of Health Science, Debre Markos University, Debre Markos, Ethiopia \\ ${ }^{2}$ Department of Human Nutrition and Food Science, College of Health Science, Debre Markos University, Debre Markos, Ethiopia \\ ${ }^{3}$ Center of Excellence in Human Nutrition, School of Human Nutrition, Food Science and Technology, Hawassa University, Ethiopia \\ ${ }^{4}$ Department of Nursing, College of Medicine and Health Science, Bahir Dar University, Bahir Dar, Ethiopia \\ ${ }^{5}$ Department of Nursing, College of Health Science, Debre Tabor University, Debre Tabor, Ethiopia
}

Correspondence should be addressed to Temesgen Getaneh; temugetaneh@gmail.com

Received 17 April 2021; Accepted 17 November 2021; Published 1 December 2021

Academic Editor: Pradeep Tyagi

Copyright (c) 2021 Temesgen Getaneh et al. This is an open access article distributed under the Creative Commons Attribution License, which permits unrestricted use, distribution, and reproduction in any medium, provided the original work is properly cited.

\begin{abstract}
Objective. Urinary tract infection (UTI) is the most common bacterial infections during pregnancy. It is associated with different maternal and neonatal adverse outcomes such as low birth weight, preterm birth, still birth, preeclampsia, maternal anemia, sepsis, and amnionitis, even when the infection is asymptomatic. However, in Ethiopia, it is represented with fragmented and inconclusive pocket studies. Therefore, this systematic review and meta-analysis is aimed at estimating the pooled prevalence of UTI and its associated factors among pregnant women in Ethiopia. Methods. PubMed/Medline, Embase, Cochrane Library, Google Scholar, and local sources were used to access eligible studies. Joanna Briggs Institute Meta-Analysis of Statistics Assessment and Review Instrument was applied for critical appraisal. Heterogeneity and publication bias were evaluated using $I^{2}$ statistic, funnel plot asymmetry, and Egger's tests. Random effect model was employed to estimate the pooled burden of UTI and its associated factors among pregnant women with its corresponding odds ratio (OR) and 95\% confidence interval (CI). Result. From all systematically searched articles, 14 studies were eligible for this analysis. The overall pooled prevalence of UTI among pregnant women in Ethiopia was 15.37\% (95\% CI: 12.54, 18.19). Family monthly income $(\mathrm{OR}=3.8$ and $95 \% \mathrm{CI}: 1.29,11.23)$, parity $(\mathrm{OR}=1.59$ and $95 \% \mathrm{CI}: 1.01,2.50)$, history of catheterization $(\mathrm{OR}=2.76$ and $95 \% \mathrm{CI}: 1.31,5.84)$, and history of UTI $(\mathrm{OR}=3.12$ and $95 \% \mathrm{CI}: 1.74,5.60)$ were factors significantly associated with UTI among pregnant women in Ethiopia. Conclusion. The overall pooled estimate of UTI among pregnant women in Ethiopia was higher compared with CDC estimation which was $8 \%$. Family monthly income $<1000 \mathrm{ETB}$, multipara, previous history of catheterization, and history of UTI were factors increased burden of UTI during pregnancy. So, strategies targeting in economic reforms, universal access of family planning, and standardized prenatal care service should be addressed to alleviate this high prevalence of UTI during pregnancy.
\end{abstract}

\section{Introduction}

The likelihood of urinary tract infections (UTIs) is increased during pregnancy due to numerous women's physiological changes [1]. The main reason is due to urinary stasis and vesicoureteral reflux caused by hormonal and mechanical changes [2]. In addition, short urethra and difficulty with hygiene due to a distended pregnant belly make UTI the commonest bacterial infectious during pregnancy which occurs in about $8 \%$ of pregnant women [3]. Furthermore, pregnant patients are considered immunocompromised UTI hosts because of the physiologic changes associated 
with pregnancy [4]. These changes increase the risk of serious infectious complications from symptomatic and asymptomatic urinary infections even in healthy pregnant women [5].

It is associated with increased risks of maternal and neonatal morbidity and mortality, even when the infection is asymptomatic [6]. If asymptomatic bacteriuria is untreated in pregnancy, the rate of subsequent UTI is approximately 25\% [6]. Even though maternal UTI has few direct fetal sequelae because fetal bloodstream infection is rare, uterine hypoperfusion due to maternal dehydration, maternal anemia, and direct bacterial endotoxin damage to the placental vasculature may cause fetal cerebral hypoperfusion [7].

It may also pose a serious health risk to a pregnant woman and developing fetus and cause of antepartum intensive care unit admission [8]. It is associated with different maternal and neonatal adverse outcomes such as low birth weight, premature labor and prematurity, still birth, preeclampsia, maternal anemia and sepsis, and amnionitis $[9,10]$. In addition, it is also associated with premature rapture of membrane and low Apgar score. This may lead to increased maternal and perinatal morbidity and mortality $[11,12]$. Furthermore, UTI during pregnancy is independently associated with intrauterine growth restriction, preeclampsia, preterm delivery, and cesarean delivery $[13,14]$. In addition to those adverse risks, it also had financial threats in which the annual health costs for UTI exceed two billion\$. Screening for these conditions in pregnant women is cost-effective, compared with treating UTI and its complication [15].

Evidences showed that pregnant women in developing countries have higher rates of UTI and its burden than developed nations $[5,16]$. Previous history of UTI, preexisting diabetes, increased parity, low socioeconomic status, immunosuppression, tobacco use, extreme maternal age, and late presentation for prenatal care are some of the risk factors [17, 18]. Among the common cause of UTI during pregnancy, Escherichia coli shared the highest while the rest was caused by different pathogens like Klebsiella pneumoniae, Proteus mirabilis, Enterobacter species, Staphylococcus saprophytic, and group B beta hemolytic streptococcus [7].

In Ethiopia, the burden of UTI among pregnant women widely varies. It ranges from $9.8 \%$ [19] to $26.6 \%$ [20]. Several fragmented individual studies were done in Ethiopia to estimate the show of the burden of UTI and its associated factors among pregnant women [19-33]. Among these studies, the lowest burden of UTI was reported in Amhara region [19] while the highest burden was occurred in Oromo regional state of Ethiopia [20]. Furthermore, sociodemographic factors like maternal age, residence, marital status, maternal educational status, monthly family income, and maternal occupation and medical and obstetric related factors like anemia, HIV status, history of UTI, history of catheterization, parity, and gestational age were some of modifiable and nonmodifiable factors stated in each fragmented studies as a potential-associated factors for UTI among pregnant women in Ethiopia [19, 21, 22, 25-27, 31, 32]. However, all of these studies were inconclusive. Currently in Ethiopia, there is no concrete evidence reporting the overall pooled prevalence of UTI and its associated factors among pregnant women. Therefore, this systematic review and meta-analysis was aimed at estimating the pooled prevalence of UTI and its associated factors among pregnant women in Ethiopia.

\section{Methods}

2.1. Searching Strategies. From Prospero, burden of UTI and its associated factors among pregnant women in Ethiopia: systematic review and meta-analysis was searched to avoid duplication. To the best of our knowledge, this is the first systematic review meta-analysis done in Ethiopia in this title. PubMed, Medline, Embase, Cochrane Library, Google Scholar, and local sources including academic and governmental institution online library were used to access included articles. In addition, the cross-references (lists of already identified articles references) were applied to retrieve studies. The key terms used for systematically searching relevant literatures were UTI, asymptomatic or symptomatic, bacteriuria, bacteria profile, prenatal, pregnancy, antenatal, associated factors, determinants, predictors, risk factors, causes, and Ethiopia. All studies on electronic databases and local sources were searched till March/2021. Then, identified articles were exported into endnote citation manager software version X7 for Windows to exclude duplicate records. The Preferred Reporting Items for Systematic Reviews and Meta-Analyses Protocols (PRISMA) checklist guidelines [34] were used to develop this systematic review and meta-analysis.

2.2. Eligibility Criteria. Study scope: all studies conducted in all regional states and administrative cities of Ethiopia on burden of UTI and its associated factors during pregnancy were included under this systematic review and metaanalysis. No restriction was applied to language, study design, study setting, and publication.

Population: all reproductive aged women (15-49 years) who were pregnant at least once were considered for this review.

Exposure: all studies explored burden of UTI and its associated factors among pregnant women in Ethiopia were included.

Outcome variable: studies which diagnosed UTI during pregnancy when their urine yielding positive cultures $\left(\geq 10^{5} \mathrm{CFU} / \mathrm{ml}\right)$ were included for this review.

After all identified studies title and abstract screened for eligibility, studies unrelated to our review title were excluded. Then, full texts of those articles related to our title and eligible were critically examined. However, those papers which did not fully access at the time of our search process were excluded after contact was attempted with the principal investigator through email at least two times. Furthermore, after reviewing full texts of all eligible studies, studies which did not report our outcome of interest and studies with poor quality as per settled criteria of reviewing the articles were excluded from the final analysis. 


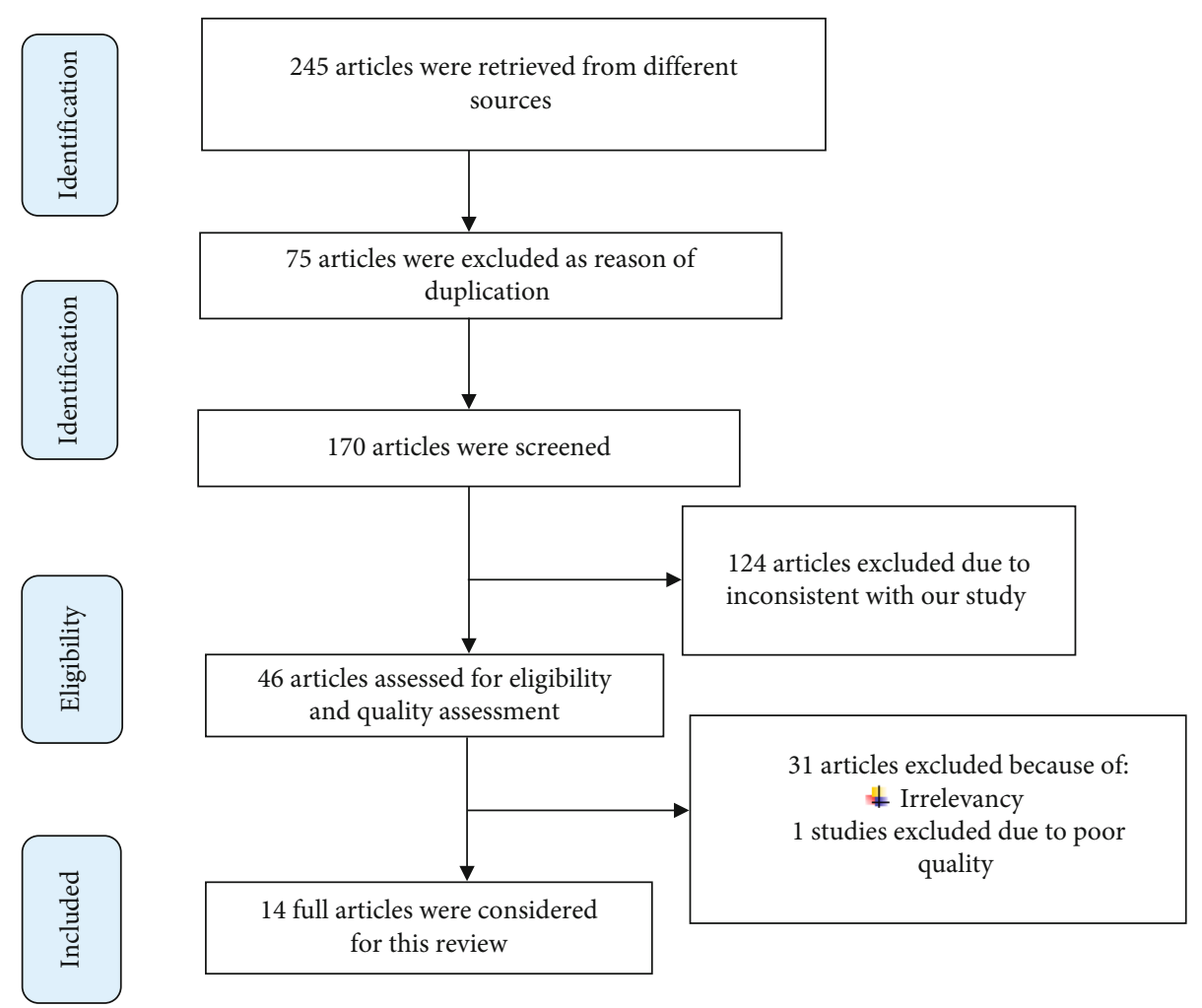

FIGURE 1: PRISMA flow diagram of included studies to estimate the pooled prevalence of UTI and its associated factors among pregnant women in Ethiopia from 2007 to 2018.

2.3. Data Extraction. Data extraction was undertaken using standardized data extraction Excel spreadsheet format prepared according to 2014 Joanna Briggs Institute Reviewers' Manual [35]. This standardized data extraction format includes the following: author name, study of region, publication year, study design, sampling technique, study setting, sample size, mean age of respondent, standard used to diagnosed UTI, response rate, and prevalence of UTI among pregnant women. Factors associated with UTI during pregnancy were also systematically extracted using cross tabulation between UTI and those potential-associated factors (including sociodemographic factors like maternal age ( $\geq 25$ yrs vs. $<25$ yrs), residence (rural vs. urban), marital status (married vs. single), maternal educational status (illiterate vs. formal education), monthly family income ( $<1000$ ETB vs. $\geq 1000$ ETB in which $1000 \mathrm{ETB}$ is equivalent with $23.8 \$$ and we have used the cut point 1000ETB because the exist data were not classified based similarly and not based on national income level classification), and maternal occupation (housewife vs. employed)) and medical and obstetric related factors like anemia (yes vs. no), HIV status (positive vs. negative), history of UTI (yes vs. no), history of catheterization (yes vs. no), parity (multipara vs. primipara and nulliparous), and gestational age (second and third trimester vs. first trimester). Disagreements between the authors were resolved by face to face discussion and consensus.

2.4. Quality Assessment. Before data extraction was handled, critical appraisal of included and eligible studies was employed using Joanna Briggs Institute Meta-Analysis of Statistics Assessment and Review Instrument (JBI-MAStARI) [35]. In the appraisal tool, randomness of subject selection, clear definition of inclusion criteria, identification and addressing for confounding factors, clear objective, and reliable measurement of outcome variable and use of appropriate statistical analysis method were included. Two independent reviewers evaluated each included individual studies critically. Disagreements between the reviewers were resolved via discussion and consensus. If not, the third reviewer was involved. Finally, those articles scored five and above were considered in this review.

We assessed and evaluated the methodological quality and risk of bias in the studies that were selected using the 10-item rating scale developed by Hoy et al. for prevalence studies [36]. Sampling, data collection, reliability and validity of study tools, case definition, and prevalence periods were included in the tool. The rating scale was categorized as having low risk of bias ("yes" answers to domain questions) or high risk of bias ("no" answers to domain questions) for each articles. Each study was assigned a score of 1 (yes) or 0 (no) for each domain, and these scores were summed to provide an overall study quality score. Scores of 8-10 were considered as having a "low risk of bias," 6-7 a "moderate risk," and 0-5 a "high risk." For the final risk of bias classification, disagreements between the reviewers were resolved via consensus.

2.5. Outcome Measurement. The primary outcome of this systematic review and meta-analysis was estimating the 
TABLE 1: Descriptive summary of 14 studies included in the meta-analysis of pooled prevalence of UTI and its associated factors among pregnant women in Ethiopia from 2007 to 2018.

\begin{tabular}{|c|c|c|c|c|c|c|c|c|c|c|c|}
\hline Author & $\begin{array}{c}\text { Publication } \\
\text { year }\end{array}$ & Region & E. coli & GNB & GPB & $\begin{array}{l}\text { Sampling } \\
\text { technique }\end{array}$ & $\begin{array}{c}\text { Mean } \\
\text { age }\end{array}$ & $\begin{array}{l}\mathrm{RR} \\
(\%)\end{array}$ & $\begin{array}{c}\text { Sample } \\
\text { size }\end{array}$ & $\begin{array}{c}\text { Prevalence } \\
(\%)\end{array}$ & $\begin{array}{l}\text { JBI } \\
\text { score }\end{array}$ \\
\hline Derese et al. & 2016 & $\begin{array}{c}\text { Dire } \\
\text { Dawa }\end{array}$ & 34.6 & 73 & 27 & Consecutive & - & $100 \%$ & 186 & 14 & 5 \\
\hline Derbie et al. & 2018 & Amhara & 11.1 & 25.9 & 74.1 & Consecutive & 26.8 & $100 \%$ & 234 & 11.5 & 5 \\
\hline Ali et al. & 2018 & Amhara & 31 & 36.2 & 63.8 & Consecutive & 26.5 & $100 \%$ & 358 & 15.6 & 6 \\
\hline Alemu et al. & 2012 & Amhara & 47.5 & 67.5 & 32.5 & SyRS & 26 & $100 \%$ & 385 & 10.4 & 8 \\
\hline Ferede et al. & 2012 & Amhara & 41.5 & 58.3 & 41.7 & Consecutive & 28 & $95 \%$ & 200 & 12 & 6 \\
\hline Tsegay et al. & Unpub & Tigray & 30 & 60 & 40 & Consecutive & 25 & $100 \%$ & 168 & 11.9 & 5 \\
\hline Taye et al. & 2018 & SNNP & 27.3 & - & - & SyRS & - & $100 \%$ & 169 & 26 & 8 \\
\hline Tadesse et al. & 2018 & Tigray & 34.6 & 64.1 & 35.9 & Consecutive & 26 & $100 \%$ & 259 & 21.2 & 7 \\
\hline Tadesse E et al. & 2014 & SNNP & 26.1 & 49 & 51 & Consecutive & 26.13 & $100 \%$ & 244 & 18.8 & 6 \\
\hline Tadesse A et al. & 2007 & Amhara & 47 & - & - & Consecutive & 26 & $96 \%$ & 173 & 9.8 & 5 \\
\hline Nisha et al. & 2015 & Oromo & 37.3 & - & - & Consecutive & 25.7 & $100 \%$ & 367 & 26.6 & 7 \\
\hline Zebene et al. & Unpub & AA & 44.4 & - & - & Consecutive & 27 & $100 \%$ & 424 & 14.9 & 5 \\
\hline Asefa et al. & 2008 & AA & 44 & 60 & 40 & Consecutive & - & $100 \%$ & 414 & 11.6 & 5 \\
\hline Emiru et al. & 2013 & Amhara & - & - & - & Consecutive & - & $100 \%$ & 367 & - & 7 \\
\hline
\end{tabular}

pooled burden of UTI among pregnant women in Ethiopia. UTI during pregnancy was diagnosed when their midstream urine sample yields positive cultures $\left(\geq 10^{5} \mathrm{CFU} / \mathrm{ml}\right)$ in either symptomatic or asymptomatic pregnant women. In addition, this review is also aimed at identifying factors associated with the pooled burden of UTI during pregnancy. Generally, sociodemographic factors like maternal age, residence, marital status, maternal educational status, monthly family income, and maternal occupation and medical and obstetric related factors like anemia, HIV status, history of UTI, history of catheterization, parity, and gestational age were identified factors to be associated with burden of UTI.

2.6. Statistical Analysis. Those data extracted using the prepared Excel spreadsheet format were imported to Stata version 14 for further analysis. The existence of heterogeneity was assessed using the Cochran's $Q$ statistic while the inverse variance $\left(I^{2}\right)$ was used to quantify it. A value at $25 \%, 50 \%$, and $75 \%$ was considered as low, moderate, and high heterogeneity across studies, respectively [37]. In addition, Egger's regression test and asymmetry funnel plot were applied to assess publication bias [38]. Furthermore, $p$ value less than 0.05 was used to declare the presence of heterogeneity across studies and publication bias. Random effect model was computed to estimate the pooled burden of UTI and its associated factors during pregnancy using forest plot diagram with their corresponding 95\% CI and OR. Moreover, subgroup analysis and metaregression were conducted to explore potential sources of heterogeneity across studies using different characteristics of the studies. Generally, the methodology part of our research may be overlapped with our previous work which was unpublished (leave for further updating and overall changing) [39].
TABLE 2: Criteria used for scoring of risk bias assessment tool of included articles for the estimation of pooled prevalence of UTI and its associated factors among pregnant women in Ethiopia from 2007 to 2018.

\begin{tabular}{lccc}
\hline Criteria & \multicolumn{3}{c}{ Bias score } \\
& Low risk & Medium risk & High risk \\
\hline Representation & 12 studies & - & 2 studies \\
Sampling & 2 studies & - & 12 studies \\
Random selection & 4 studies & - & 10 studies \\
Nonresponse bias & 14 studies & - & - \\
$\begin{array}{l}\text { Data collection } \\
\text { Case definition }\end{array}$ & 14 studies & - & - \\
Reliability and validity & 14 studies & - & - \\
of study tool & 10 studies & - & 4 studies \\
$\begin{array}{l}\text { Method of data collection } \\
\text { Prevalence period }\end{array}$ & 10 studies & - & 4 studies \\
$\begin{array}{l}\text { Numerator and } \\
\text { denominator }\end{array}$ & 14 studies & - & - \\
Summary assessment & 10 studies & - & - \\
\hline & & - & 4 studies \\
\hline
\end{tabular}

\section{Results}

3.1. Explanation for Original Studies. From medical-related electronic databases and local sources, a total of 245 articles were accessed. 75 articles were excluded because of duplication, and 124 were removed as reason of inconsistent with our review. Then, the remaining 46 studies were assessed and screened based on eligibility criteria. After screening, 31 articles were removed because of irrelevancy. Using JBIMAStARI, 15 full articles were critical appraised. After critical appraisal, one articles [40] was excluded because of low quality scored. Finally, 14 studies were included for 


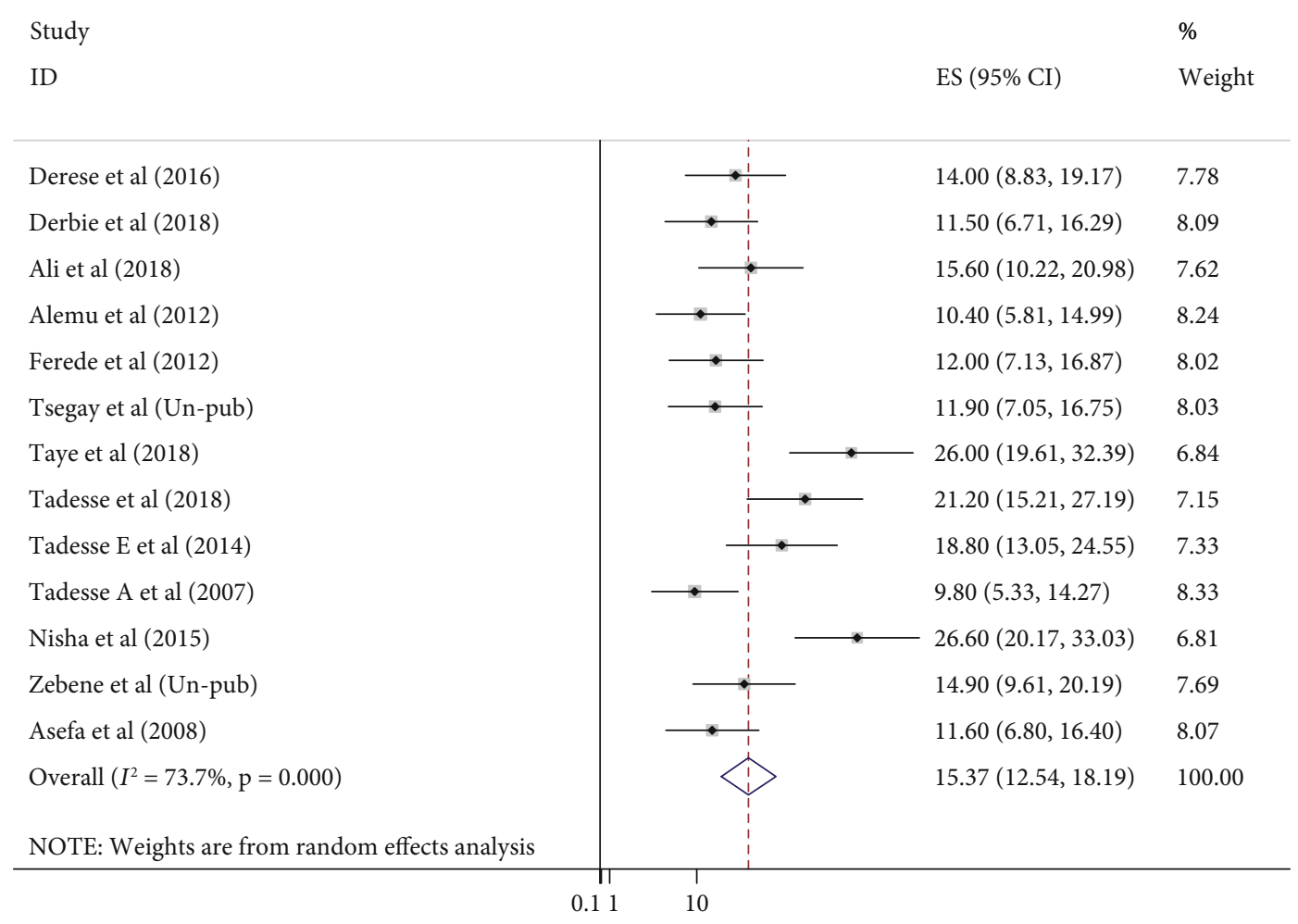

FIGURE 2: Forest plot of the pooled prevalence of UTI and its associated factors among pregnant women in Ethiopia from 2007 to 2018.

estimating the pooled prevalence of UTI and its associated factors during pregnancy in Ethiopia (Figure 1).

Among 14 eligible and included articles, 12 of them were published studies from 2007 to 2018 whereas the remaining two studies were unpublished. All included articles were conducted using institutional based cross sectional study design. Around $86 \%$ of the included studies (12 studies) used consecutive sampling technique while $14 \%$ of them (two studies) used systematic random sampling technique. All studies considered UTI among pregnant women when their urine sample yielding positive cultures $\left(\geq 10^{5} \mathrm{CFU} / \mathrm{ml}\right)$ and had $95 \%$ and above of response rate. A total of 3,948 reproductive age (15-49) women who have at least one pregnancy were included to estimate the pooled prevalence of UTI and its associated factors during pregnancy in Ethiopia.

From the included studies, six articles were in Amhara regional state $[19,21,22,26,28,29]$ whereas Tigray, SNNP and AA shared two articles for each [23, 27, 30-33] while Oromo [20] and Dire Dawa [25] contribute only a single study for each. According to JBI-MAStARI critical appraisal, each included individual articles scored a minimum of five to maximum of 8 out of 9 . In addition, nearly $55 \%$ of UTI during pregnancy was caused by Gram-negative bacteria (GNB) while the remaining $45 \%$ of UTI was caused by Gram-positive bacteria. Furthermore, Escherichia coli is responsible for the cause of around 35\% of UTI during pregnancy (Table 1).

In regarding risk bias assessment, $10(71.5 \%)$ studies had high quality scores and 4 (28.5\%) had low quality scores (Table 2). Representation and case-definition biases were the most commonly noted. To determine the influence of

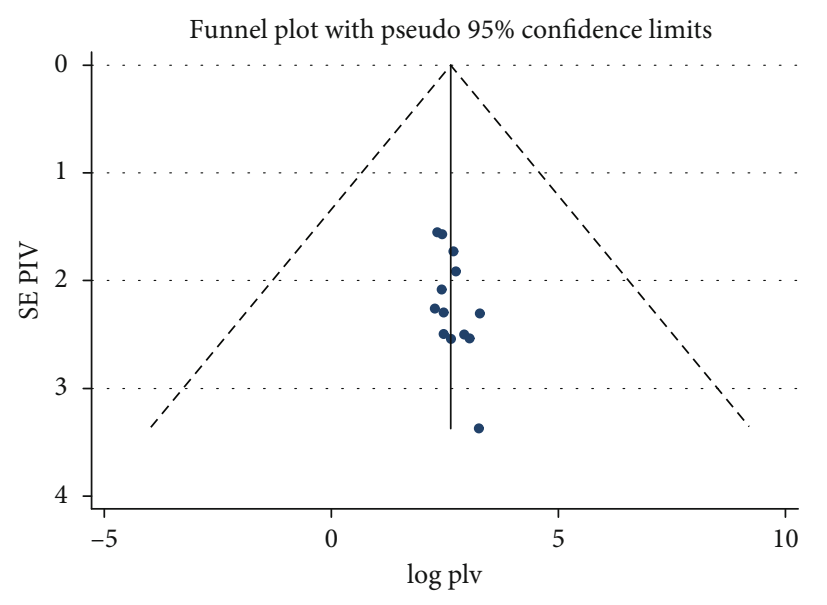

FIGURE 3: Meta funnel presentation of the pooled prevalence of UTI and its associated factors among pregnant women in Ethiopia from 2007 to 2018.

low methodological quality/high risk of bias on our estimates of pooled prevalence, we estimated pooled prevalence without the low-quality studies. The confidence intervals of our estimates of pooled prevalence with and without these studies overlapped, indicating no significant difference between them. These results suggest that the majority of the primary study authors have met high-quality standards. This lends credibility to our findings.

3.2. Prevalence of UTI among Pregnant Women in Ethiopia. The estimating pooled prevalence of UTI among pregnant 
TABLE 3: Subgroup analysis which describes pooled burden of UTI and its associated factors among pregnant women in Ethiopia from 2007 to 2018 .

\begin{tabular}{|c|c|c|c|c|c|c|}
\hline Subgroup & & No. of studies & Prevalence (95\% CI) & Heterogeneity statistics & $I^{2}$ & $p$ value \\
\hline \multirow{5}{*}{ Region } & Amhara & 5 & $11.61(9,13)$ & 3.03 & 0.0 & 0.553 \\
\hline & Tigray & 2 & $16.37(7,25)$ & 5.59 & 82.1 & 0.018 \\
\hline & SNNPR & 2 & $22.26(15,29)$ & 2.70 & 62.9 & 0.101 \\
\hline & AA & 2 & $13.09(9,16)$ & 0.82 & 0.0 & 0.366 \\
\hline & Others & 2 & $20.14(7,32)$ & 8.95 & 88.1 & 0.003 \\
\hline \multirow{3}{*}{ Publication year } & Before 2015 & 5 & $12.19(9,15)$ & 6.79 & 41.1 & 0.143 \\
\hline & 2015 \& above & 6 & $18.88(13,23)$ & 24.13 & 79.3 & $<0.001$ \\
\hline & Unpublished & 2 & $13.27(9,16)$ & 0.67 & 0.0 & 0.412 \\
\hline \multirow{2}{*}{ Sampling technique } & Consecutive & 11 & $14.92(12,17)$ & 30.01 & 66.7 & 0.001 \\
\hline & Systematic random & 2 & $18.03(2,33)$ & 15.12 & 92.1 & $<0.001$ \\
\hline \multirow{2}{*}{ Sample size } & $<250$ & 7 & $14.51(10,18)$ & 22.00 & 72.1 & 0.001 \\
\hline & $>250$ & 6 & $16.4(11,21)$ & 22.25 & 77.5 & $<0.001$ \\
\hline
\end{tabular}

women in Ethiopia was 15.37\% (95\% CI: 12.54, 18.19) (Figure 2). The Cochran's $Q$ statistic evidenced the presence of moderate statistically significant level of heterogeneity $\left(I^{2}=73.7 \%, p<0.001\right)$ which indicated that the use of random model effect to estimate the pooled prevalence of UTI and its associated factors among pregnant women was appropriate. Therefore, to identify potential sources of heterogeneity, subgroup analysis and metaregression were undertaken. In addition, publication bias was checked using asymmetric funnel plot and Egger's regression test. Both symmetric funnel plot and Egger's test evidenced, and publication bias was not observed in this review (symmetric funnel plot (Figure 3) and Egger's test $p$ value $=0.069(95 \%$ CI: 0.201-1.734)).

3.3. Subgroup Analysis. Subgroup analysis was also performed using different study characteristics. In regarding to regional burden, more than one-fifth of pregnant women were diagnosed with UTI in SNNP region with prevalence of $22.2 \%(95 \%$ CI: 15,29$)$. The lowest overall prevalence of UTI among pregnant women was recorded in Amhara regional state of Ethiopia which was 11.6\% (95\% CI: 9, 13) while in Tigray $16.3 \%$ (95\% CI: 7,25$)$ and in AA $13.09 \%$ (95\% CI: 9, 16) of pregnant women were diagnosed with UTI.

In addition, studies published before 2015 reported 12.2\% (95\% CI: 9, 15) of UTI among pregnant women whereas studies published after 2015 and after evidenced that $18.8 \%(95 \%$ CI: 13,23$)$ of UTI among pregnant women. The pooled prevalence of UTI among studies having less 250 sample size was $14.5 \%$ (95\% CI: 10, 18) whereas studies with sample size of more than 250 indicated $16.4 \%$ (95\% CI: 11 , 21) of UTI among pregnant women (Table 3 ).

3.4. Metaregression. In order to identify potential source of heterogeneity, metaregression was done in addition to subgroup analysis using both continuous and categorical study characteristics. However, none of these variables were found to be statistically significant (Table 4).
TABLE 4: Metaregression for the included studies to identify source of heterogeneity for the pooled prevalence of UTI and its associated factors among pregnant women in Ethiopia from 2007 to 2018.

\begin{tabular}{lcc}
\hline Variables & Coefficients & $p$ value \\
\hline Study year & -0.126 & 0.078 \\
Sample size & -0.019 & 0.602 \\
Mean age & 0.91 & 0.532 \\
Region & & \\
$\quad$ Amhara & -8.60 & 0.056 \\
SNNPR & 1.53 & 0.758 \\
Tigray & -3.94 & 0.424 \\
AA & -7.25 & 0.144 \\
\hline
\end{tabular}

3.5. Factors Associated with UTI among Pregnant Women in Ethiopia. In addition to estimating the pooled prevalence of UTI among pregnant women in Ethiopia, this review also examined the association of different sociodemographic and medical and obstetric related factors with UTI during pregnancy. Maternal age, residence, marital status, maternal educational status, monthly family income, maternal occupation, anemia, HIV status, history of UTI, history of catheterization, parity, and gestational age were among the factors evaluated. But only monthly family income, history of UTI and catheterization, and parity were significantly associated with the pooled prevalence of UTI among pregnant women (Figures 4-6).

This review showed that monthly family income was significantly associated with UTI among pregnant women in which its association was stated in 6 of the included studies. The odds of UTI among pregnant women with family monthly income less than 1000ETB were 3.8 times higher than their counterparts $(\mathrm{OR}=3.8,95 \% \mathrm{CI}: 1.29,11.23)$ (Figure 4).

To evaluate the association between histories of UTI with current UTI among pregnant women, 8 studies were 


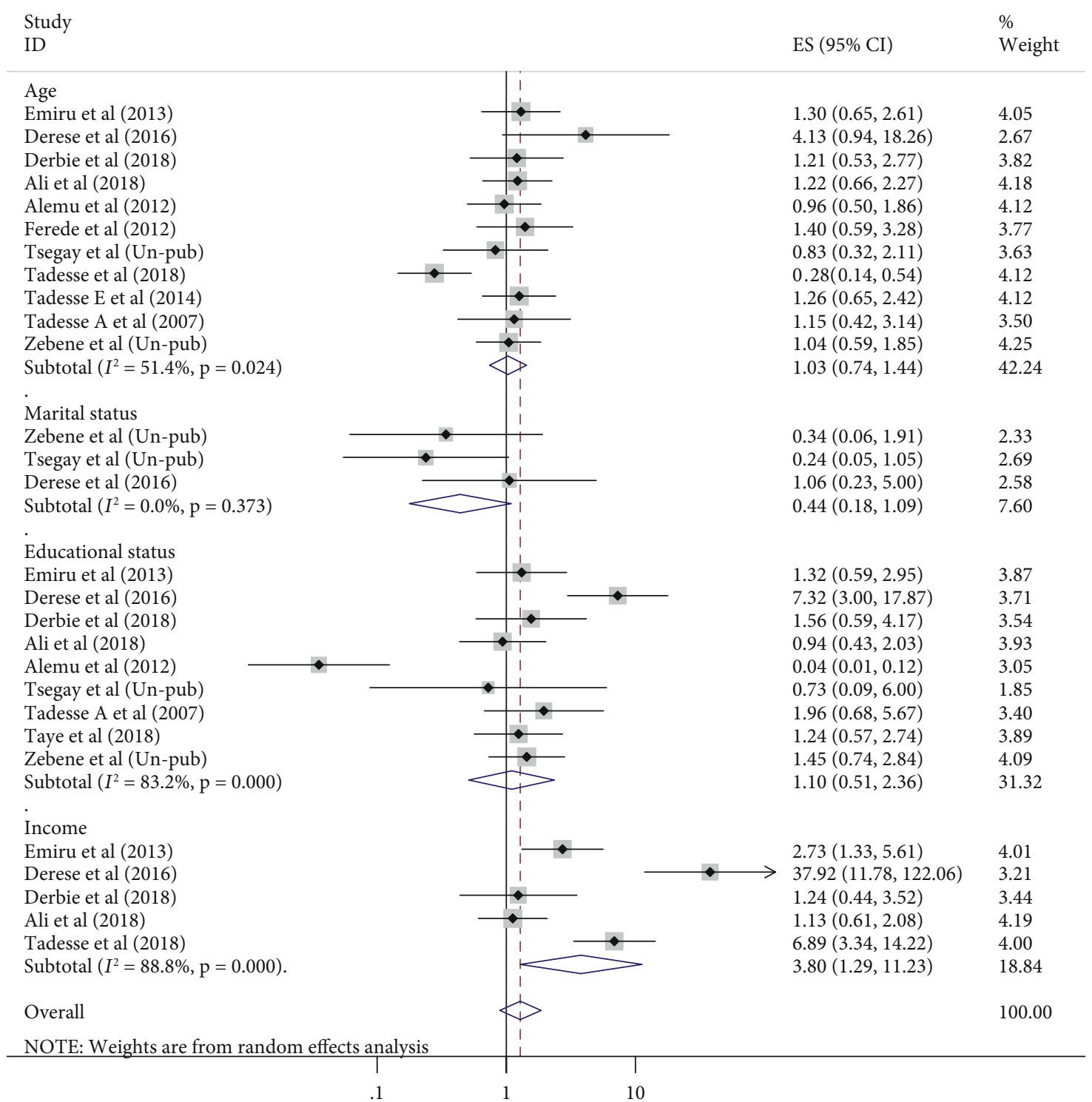

FIGURE 4: Forest plots which describe association between maternal age, marital status, education and income, and UTI among pregnant women in Ethiopia from 2007 to 2018.

included. The analysis of these studies indicated that the likelihood of UTI among pregnant women who had history of UTI was 3.12 times higher than their counterparts $(\mathrm{OR}=3.12$, 95\% CI: 1.74, 5.60) (Figure 5).

History of catheterization was another significantly associated factor with pooled prevalence of UTI among pregnant women. Its connection with UTI was cited in 7 of included studies. Pregnant women who had history of catheterization were 2.76 times more likely to develop UTI than pregnant women who had no history of catheterization $(\mathrm{OR}=2.76$, $95 \%$ CI: $1.31,5.84)$. Once more, parity was also another factor associated with UTI during pregnancy in which its relation was listed in 8 of the original included articles. The odds of UTI among multipara pregnant women were 1.6 times higher than primipara and nulliparous pregnant women (OR $=1.59,95 \% \mathrm{CI}: 1.01,2.50)$ (Figure 6). Maternal age, residence, marital status, maternal educational status, maternal occupation, anemia, HIV status, and gestational age were not associated with pooled burden of UTI among pregnant women in Ethiopia.

\section{Discussion}

This is the first systematic review and meta-analysis done in Ethiopia to estimate the pooled prevalence of UTI and its associated factors among pregnant. According to this review, the overall pooled prevalence of UTI among pregnant women in Ethiopia was $15.37 \%$ (95\%, CI: 12.54, 18.19). This figure is higher than prevalence of UTI during pregnancy estimated by CDC which was $8 \%$. The possible explanation may be in our country, early initiation of antenatal care service was low. So, there should have early detection and identification of risk factors. In addition, majority of pregnant women will not get health education given for pregnant 


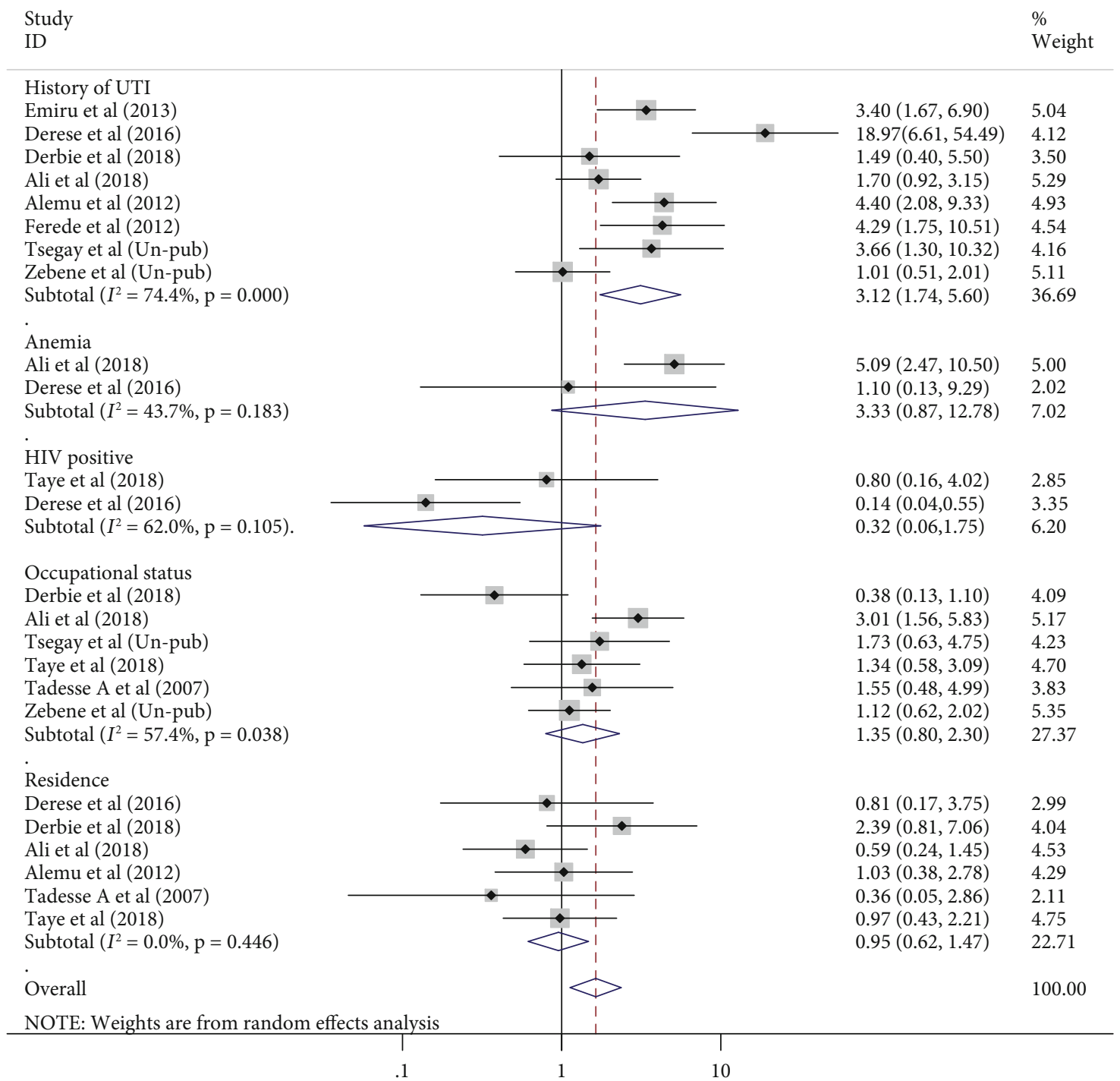

FIGURE 5: Forest plots which describe association of history of UTI, anemia, HIV status, occupation, and residence with UTI among pregnant women in Ethiopia from 2007 to 2018.

women for infection prevention due to delay initiation. Furthermore, majority of our population were rural with no formal education which in turn characterize with less level of awareness on infection prevention and healthseeking behavior and lower economic level which will be a significant factor in many aspects.

Therefore, this incompatible and high evidence of the pooled prevalence of UTI during pregnancy in Ethiopia should be taken as a warning and baseline evidence for healthy policy makers, program planner, and implementers to improve maternal and neonatal outcomes. This figure is in line with findings of studies held in Sudan and Tanzania [41, 42]. But the finding is much higher than systematic review done in Iran and CDC estimation and studies conducted in Bangladesh, South Africa, Cameroon, and Saudi Arabia [3, 43-47]. However, the figure of this review is much lower than result of studies conducted in Egypt, Pakistan, and Libya which was 32\%, 23.9\%, and $30 \%$, respectively [48-50].
In addition to estimating the pooled burden of UTI, this meta-analysis also identified factors to be associated with the pooled prevalence of UTI among pregnant women in Ethiopia. Among factors evaluated, only family monthly income, parity, history of catheterization, and history of UTI were significantly associated factors with the overall pooled prevalence of UTI among pregnant women in Ethiopia.

The odds of UTI among pregnant women whose family income was less than 1000ETB were higher than their counterparts. This figure is in line with studies conducted in Egypt and Pakistan [2, 49]. This could be evidenced that pregnant women with low economic status were majorly exposed to malnutrition in turn had impact on immunity status. Parity was also another factor significantly that increased the odds of UTI during pregnancy. The likelihood of UTI was higher among multipara pregnant women than primipara and nulliparous pregnant women. This finding is line with result of studies conducted in Nigeria, Iran, and Pakistan $[2,51,52]$. 


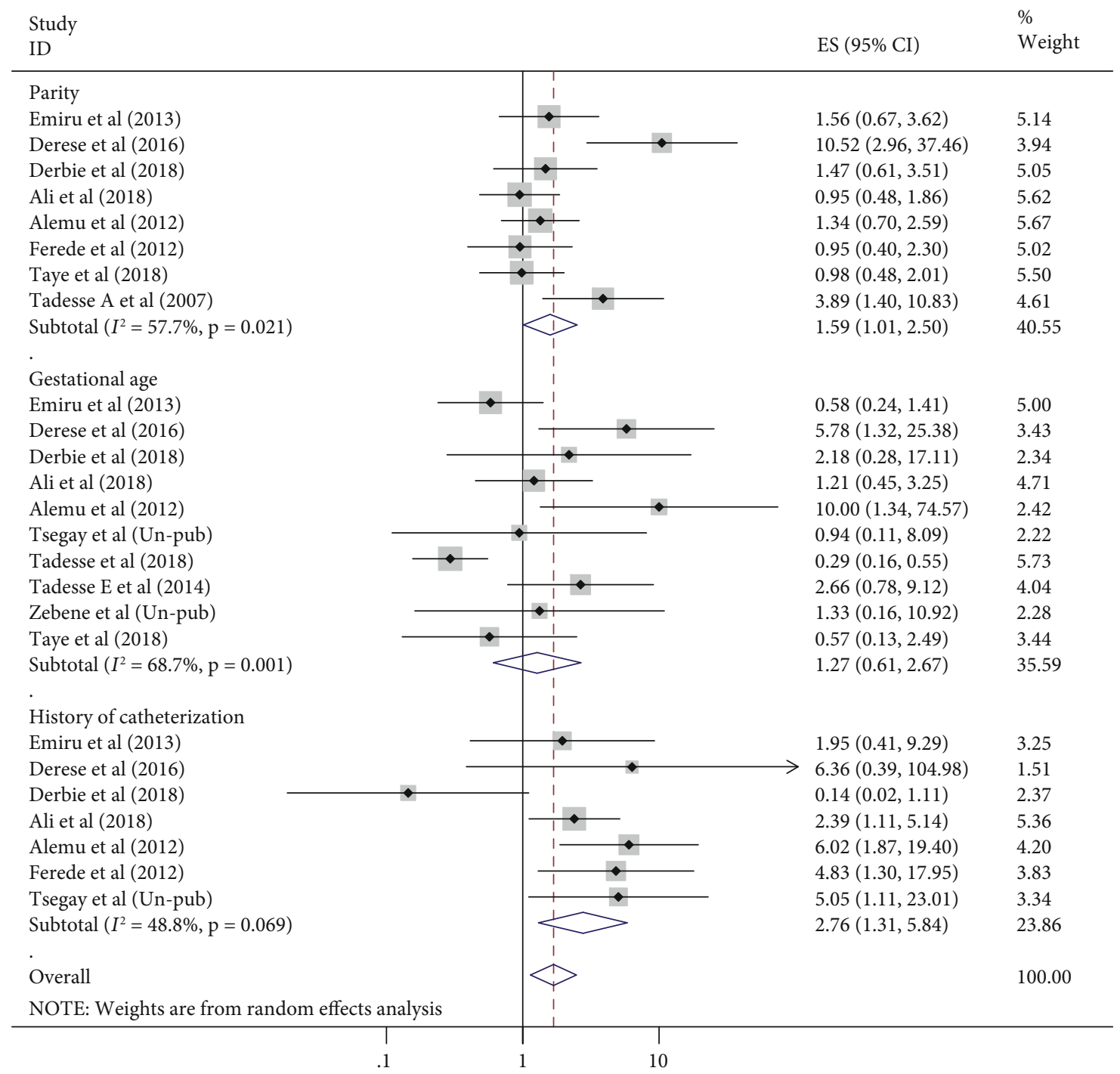

FIGURE 6: Forest plots which describe association of history of catheterization, parity, and gestational age with UTI among pregnant women in Ethiopia from 2007 to 2018.

The association between multiparity and UTI is due to profound physiologic changes affecting the entire urinary tract during pregnancy has a significant impact on the natural history of UTI during gestation [53]. These changes vary from patient to patient and are more likely to occur in women who have pregnancies in rapid succession. Furthermore, the anatomical relationship of the female urethra to the vagina makes it liable to trauma during childbirth [54].

In addition, previous history of UTI was a significant factor associated with UTI among pregnant women. The odds of UTI among pregnant women who had history of UTI were higher than their counterparts. This result was in agreement with studies conducted in Pakistan, Qatar, Philippines [2, 53, 55], and Saudi Arabia [44]. This may be evidenced by the risk of developing resistance strains increased among pregnant women who had previous history of UTI.

Once more, this review revealed that the odds of UTI among pregnant women who had previous history of cathe- terization were higher than their counterparts. This finding is supported with studies conducted in Korea, Israel, and Nigeria [56-58]. This might be due to catheterization is an invasive procedure and could induce urethral mucosa injury. It can also lead to the introduction of bacterial organism to the bladder which induces haematogenous bacterial spread due to failure in infection prevention or poor aseptic technique which can be responsible for recurrent UTI $[4,59]$. Generally, this concrete evidences will have positive influential impact to improve maternal and neonatal health in terms of alleviating this high burden of UTI. In addition, this evidence will be used as baseline for healthy policy makers, planners, and implementers.

4.1. Study Limitation. All included studies were crosssectional study design in which the result might potentially be affected by confounding variables and difficult to establish temporal relationship between the outcome and exposure variables. In addition, the meta-analysis did not include all 
regions and administrative city which only include four regions and two administrative city of the country. Therefore, further country-based studies to assess other confounding factors related to health service-related factors in terms of quality, health policy factors, and health caregiverrelated factors are recommended.

\section{Conclusion}

The overall pooled estimate of UTI among pregnant women in Ethiopia was higher than Communicable Disease Control (CDC) estimation which was $8 \%$. Both sociodemographic, medical, and obstetric related factors which can be prevented affect the pooled prevalence of UTI among pregnant women. Family monthly income $<1000$ ETB, multipara, previous history of catheterization, and history of UTI were factors increased burden of UTI during pregnancy. These findings indicated the need for interventions. So, strategies targeting in economic reforms and universal access of family planning were needed. In addition, standardized prenatal care service as recommended to identify those risk factors early should be addressed by the Ministry of Health and its stakeholders to alleviate this high prevalence of UTI during pregnancy and its complications.

\section{Abbreviations}

AA: Addis Ababa

CDC: Communicable Disease Control

SNNP: South Nation and Nationality peoples

UTI: Urinary tract infection

WHO: World Health Organization.

\section{Data Availability}

Data will be available from the corresponding author upon reasonable request.

\section{Conflicts of Interest}

The authors have declared that there are no competing interests.

\section{Authors' Contributions}

TG developed the protocol, and TG, AN, and GD involved in the design, selection of study, data extraction, statistical analysis, and developing the initial drafts of the manuscript and quality assessment. TG, AN, GD, and MD prepared and revised subsequent drafts as well as prepared the final draft of the manuscript. Finally, all the authors read and approved the final draft of the manuscript.

\section{References}

[1] J. B. Hill, J. S. Sheffield, D. D. McIntire, and G. D. Wendel, "Acute pyelonephritis in pregnancy," Obstetrics \& Gynecology, vol. 105, no. 1, pp. 18-23, 2005.

[2] G. Haider, N. Zehra, A. A. Munir, and A. Haider, "Risk factors of urinary tract infection in pregnancy," JPMA The Journal of the Pakistan Medical Association, vol. 60, no. 3, pp. 213-216, 2010.

[3] J. E. Delzell Jr. and M. LeFevre, "Urinary tract infections during pregnancy," American Family Physician, vol. 61, no. 3, pp. 713-721, 2000.

[4] O. Storme, J. Tiran Saucedo, A. Garcia-Mora, M. DehesaDávila, and K. G. Naber, "Risk factors and predisposing conditions for urinary tract infection," Therapeutic Advances in Urology, vol. 11, p. 175628721881438, 2019.

[5] K. Kalinderi, D. Delkos, M. Kalinderis, A. Athanasiadis, and I. Kalogiannidis, "Urinary tract infection during pregnancy: current concepts on a common multifaceted problem," Journal of Obstetrics and Gynaecology, vol. 38, no. 4, pp. 448453, 2018.

[6] T. M. Hooton, "Uncomplicated urinary tract infection," New England Journal of Medicine, vol. 366, no. 11, pp. 1028-1037, 2012.

[7] A. P. Glaser and A. J. Schaeffer, "Urinary tract infection and bacteriuria in pregnancy," Urologic Clinics, vol. 42, no. 4, pp. 547-560, 2015.

[8] S. R. Dimetry, H. M. El-Tokhy, N. M. Abdo, M. A. Ebrahim, and M. Eissa, "Urinary tract infection and adverse outcome of pregnancy," The Journal of the Egyptian Public Health Association, vol. 82, no. 3-4, pp. 203-218, 2007.

[9] L. Yan, Y. Jin, H. Hang, and B. Yan, "The association between urinary tract infection during pregnancy and preeclampsia: a meta-analysis," Medicine, vol. 97, no. 36, p. e12192, 2018.

[10] A. W. Valkenburg-van den Berg, A. J. Sprij, F. W. Dekker, P. J. Dörr, and H. H. Kanhai, "Association between colonization with group BStreptococcusand preterm delivery: a systematic review," Acta Gynecologica Scandinavica, vol. 88, no. 9, pp. 958-967, 2009.

[11] N. M. Gilbert, V. P. O’brien, S. Hultgren, G. Macones, W. G. Lewis, and A. L. Lewis, "Urinary tract infection as a preventable cause of pregnancy complications: opportunities, challenges, and a global call to action," Global advances in health and medicine, vol. 2, no. 5, pp. 59-69, 2013.

[12] B. M. Kazemier, F. N. Koningstein, C. Schneeberger et al., "Maternal and neonatal consequences of treated and untreated asymptomatic bacteriuria in pregnancy: a prospective cohort study with an embedded randomised controlled trial," The Lancet Infectious Diseases, vol. 15, no. 11, pp. 1324-1333, 2015.

[13] E. Mazor-Dray, A. Levy, F. Schlaeffer, and E. Sheiner, "Maternal urinary tract infection: is it independently associated with adverse pregnancy outcome?," The Journal of Maternal-Fetal \& Neonatal Medicine, vol. 22, no. 2, pp. 124-128, 2009.

[14] L. A. Schieve, A. Handler, R. Hershow, V. Persky, and F. Davis, "Urinary tract infection during pregnancy: its association with maternal morbidity and perinatal outcome," American Journal of Public Health, vol. 84, no. 3, pp. 405-410, 1994.

[15] ACOG, Guidelines for Perinatal Care, Am Acad Pediatrics, 2012.

[16] N. S. Whitehead, W. Callaghan, C. Johnson, and L. Williams, "Racial, ethnic, and economic disparities in the prevalence of pregnancy complications," Maternal and Child Health Journal, vol. 13, no. 2, p. 198, 2009.

[17] J. R. Alvarez, A. J. Fechner, S. F. Williams, V. L. Ganesh, and J. J. Apuzzio, "Asymptomatic bacteriuria in pregestational diabetic pregnancies and the role of group B Streptococcus," American Journal of Perinatology, vol. 27, no. 3, pp. 231-234, 2010. 
[18] D. A. Wing, M. J. Fassett, and D. Getahun, "Acute pyelonephritis in pregnancy: an 18-year retrospective analysis," American Journal of Obstetrics and Gynecology, vol. 210, no. 3, pp. 219.e1-219.e6, 2014.

[19] A. Tadesse, M. Negash, and L. Ketema, "Asymtomatic bacteriuria in pregnancy: assesment of prevlence, microbial agents and ther antimicrobial sensitivty pattern in Gondar Teaching Hospital, north west Ethiopia," Ethiopian Medical Journal, vol. 45, no. 2, pp. 143-149, 2007.

[20] A. K. Nisha, A. E. Etana, and H. Tesso, "Prevalence of asymptomatic bacteriuria during pregnancy in Adama city, Ethiopia," Int J Microbiol Immunol Res, vol. 3, pp. 58-63, 2015.

[21] A. Alemu, F. Moges, Y. Shiferaw et al., "Bacterial profile and drug susceptibility pattern of urinary tract infection in pregnant women at University of Gondar Teaching Hospital, Northwest Ethiopia," BMC Research Notes, vol. 5, no. 1, p. ???, 2012.

[22] I. E. Ali, T. Gebrecherkos, M. Gizachew, and M. A. Menberu, "Asymptomatic bacteriuria and antimicrobial susceptibility pattern of the isolates among pregnant women attending Dessie referral hospital, Northeast Ethiopia: a hospital-based cross-sectional study," Turkish Journal of Urology, vol. 44, no. 3, pp. 251-260, 2018.

[23] A. Assefa, D. Asrat, Y. Woldeamanuel, A. Abdella, and T. Melesse, "Bacterial profile and drug susceptibility pattern of urinary tract infection in pregnant women at Tikur Anbessa Specialized Hospital Addis Ababa, Ethiopia," Ethiopian Medical Journal, vol. 46, no. 3, pp. 227-235, 2008.

[24] T. Demilie, G. Beyene, S. Melaku, and W. Tsegaye, "Diagnostic accuracy of rapid urine dipstick test to predict urinary tract infection among pregnant women in Felege Hiwot Referral Hospital, Bahir Dar, North West Ethiopia," BMC Research Notes, vol. 7, no. 1, p. ???, 2014.

[25] B. Derese, H. Kedir, Z. Teklemariam, F. Weldegebreal, and S. Balakrishnan, "Bacterial profile of urinary tract infection and antimicrobial susceptibility pattern among pregnant women attending at Antenatal Clinic in Dil Chora Referral Hospital, Dire Dawa, Eastern Ethiopia," Therapeutics and Clinical Risk Management, vol. 12, p. 251, 2016.

[26] T. Emiru, G. Beyene, W. Tsegaye, and S. Melaku, "Associated risk factors of urinary tract infection among pregnant women at Felege Hiwot Referral Hospital, Bahir Dar, North West Ethiopia," BMC Research Notes, vol. 6, no. 1, p. ???, 2013.

[27] T. Ephrem, Bacterial profile and drug susceptibility pattern of urinary tract infection in pregnant women attending antenatal care at Mekelle Hospital, Mekelle, Northern Ethiopia. Un-publish, Addis Aababa University, 2014.

[28] G. Ferede, G. Yismaw, Y. Wondimeneh, and Z. Sisay, "The prevalence and antimicrobial susceptibility pattern of bacterial uropathogens isolated from pregnant women," Eur J Exp Biol, vol. 2, no. 5, pp. 1497-1502, 2012.

[29] A. D. Habteyohannes, D. Mekonnen, E. Abate, S. Tadesse, T. Birku, and F. Biadglegne, "Bacterial isolates and their current drug susceptibility profile from urine among asymptomatic pregnant women attending at a Referral Hospital, Northwest Ethiopia; cross-sectional study," Ethiopian Journal of Reproductive Health, vol. 10, no. 2, pp. 10-10, 2018.

[30] E. Tadesse, M. Teshome, Y. Merid, B. Kibret, and T. Shimelis, "Asymptomatic urinary tract infection among pregnant women attending the antenatal clinic of Hawassa Referral Hospital, Southern Ethiopia," BMC Research Notes, vol. 7, no. 1, p. $155,2014$.
[31] S. Tadesse, T. Kahsay, G. Adhanom, G. Kahsu, H. Legese, and A. Derbie, "Prevalence, antimicrobial susceptibility profile and predictors of asymptomatic bacteriuria among pregnant women in Adigrat General Hospital, Northern Ethiopia," BMC Research Notes, vol. 11, no. 1, p. 740, 2018.

[32] S. Taye, M. Getachew, Z. Desalegn, A. Biratu, and K. Mubashir, "Bacterial profile, antibiotic susceptibility pattern and associated factors among pregnant women with Urinary Tract Infection in Goba and Sinana Woredas, Bale Zone, Southeast Ethiopia," BMC Research Notes, vol. 11, no. 1, p. 799, 2018.

[33] W. Zebene, Urinary Tract Infection, Drug Resistance Profile and Fetal Outcomes among Pregnant Women in Two Health Centers and Tikur Anbessa Specialized Hospital, Addis Ababa, Addis Ababa University, Ethiopia, 2019.

[34] A. Liberati, D. G. Altman, and J. Tetzlaff, “The PRISMA statement for reporting systematic reviews and meta-analyses of studies that evaluate health care interventions: explanation and elaboration," Journal of Clinical Epidemiology, vol. 62, no. 10, pp. e1-e34, 2009.

[35] Z. Munn, S. Moola, K. Lisy, and D. Riitano, The Joanna Briggs institute reviewers' manual. The systematic review of prevalence and incidence data, The Joanna Briggs Institute, Adelaide, 2014.

[36] D. Hoy, P. Brooks, A. Woolf et al., "Assessing risk of bias in prevalence studies: modification of an existing tool and evidence of interrater agreement," Journal of Clinical Epidemiology, vol. 65, no. 9, pp. 934-939, 2012.

[37] T. B. Huedo-Medina, J. Sánchez-Meca, F. Marín-Martínez, and J. Botella, "Assessing heterogeneity in meta-analysis: Q statistic or ${ }^{\mathrm{I}^{2}}$ index?," Psychological Methods, vol. 11, no. 2, pp. 193-206, 2006.

[38] G. Rendina-Gobioff, Detecting publication bias in random effects meta-analysis, An empirical comparison of statistical methods, 2006.

[39] T. Getaneh, A. Negesse, and J. Chane, Premarital sexual practice and its associated factors in Ethiopia, A systematic review and meta-analysis, 2019.

[40] S. Gebre-Selassie, "Asymptomatic bacteriuria in pregnancy: epidemiological, clinical and microbiological approach," Ethiopian Medical Journal, vol. 36, no. 3, pp. 185-192, 1998.

[41] A. Masinde, B. Gumodoka, A. Kilonzo, and S. Mshana, "Prevalence of urinary tract infection among pregnant women at Bugando Medical Centre, Mwanza, Tanzania," Tanzania Journal of Health Research, vol. 11, no. 3, pp. 154-159, 2009.

[42] H. Z. Hamdan, A. H. M. Ziad, S. K. Ali, and I. Adam, "Epidemiology of urinary tract infections and antibiotics sensitivity among pregnant women at Khartoum North Hospital," Annals of Clinical Microbiology and Antimicrobials, vol. 10, no. 1, p. ???, 2011.

[43] M. Azami, Z. Jaafari, M. Masoumi et al., "The etiology and prevalence of urinary tract infection and asymptomatic bacteriuria in pregnant women in Iran: a systematic review and meta-analysis," BMC Urology, vol. 19, no. 1, p. 43, 2019.

[44] H. S. Faidah, A. M. Ashshi, G. A. Abou El-Ella, A. K. AlGhamdi, and A. M. Mohamed, "Urinary tract infections among pregnant women in Makkah, Saudi Arabia," Biomedical And Pharmacology Journal, vol. 6, pp. 1-7, 2013.

[45] M. N. Uddin and T. Khan, "Prevalence of urinary tract infection among pregnant women at Ibrahim Iqbal Memorial Hospital, Chandanaish, Bangladesh," Am J Clin Med Res, vol. 4, no. 3, pp. 47-51, 2016. 
[46] F. D. P. Siemefo Kamgang, H. C. Maise, and J. Moodley, "Pregnant women admitted with urinary tract infections to a public sector hospital in South Africa: are there lessons to learn?," Southern African Journal of Infectious Diseases, vol. 31, no. 3, pp. 79-83, 2016.

[47] C. T. Nguefack, C. O. Ebongue, C. N. Chokotheu, C. E. Ewougo, T. N. Njamen, and E. Mboudou, "Clinical presentation, risk factors and pathogens involved in bacteriuria of pregnant women attending antenatal clinic of 3 hospitals in a developing country: a cross sectional analytic study," BMC Pregnancy and Childbirth, vol. 19, no. 1, p. 143, 2019.

[48] M. Sheikh, Incidence of Urinary Tract Infection during Pregnancy, 2000.

[49] H. M. Shaheen, T. M. Farahat, and H. NAE-H, "Prevalence of urinary tract infection among pregnant women and possible risk factors," Menoufia Medical Journal, vol. 29, no. 4, p. 1055, 2016.

[50] M. Tamalli, S. Bioprabhu, and M. Alghazal, "Urinary tract infection during pregnancy at Al-khoms, Libya," Int J Med Med Sci, vol. 3, no. 5, pp. 455-459, 2013.

[51] I. Okonko, L. Ijandipe, O. Ilusanya et al., "Incidence of urinary tract infection (UTI) among pregnant women in Ibadan, South-Western Nigeria," African Journal of Biotechnology, vol. 8 , no. 23, 2009.

[52] K. Enayat, F. Fariba, and N. Bahram, "Asymptomatic bacteriuria among pregnant women referred to outpatient clinics in Sanandaj, Iran," International Braz J Urol, vol. 34, no. 6, pp. 699-707, 2008.

[53] P. Nandy, A. Thakur, and C. Ray, "Characterization of bacterial strains isolated through microbial profiling of urine samples," On Line J Biol Sci, vol. 7, no. 1, pp. 44-51, 2007.

[54] A. Kolawole, O. Kolawole, Y. Kandaki-Olukemi, S. Babatunde, K. Durowade, and C. Kolawole, "Prevalence of urinary tract infections (UTI) among patients attending Dalhatu Araf Specialist Hospital, Lafia, Nasarawa state, Nigeria," International journal of medicine and medical sciences, vol. 1, no. 5, pp. 163-167, 2009.

[55] M. T. Aseel, F. M. Al-Meer, M. G. Al-Kuwari, and M. F. S. Ismail, "Prevalence and predictors of asymptomatic bacteriuria among pregnant women attending primary health care in Qatar," WORLD FAMILY MEDICINE, vol. 7, no. 4, pp. 10-13, 2009.

[56] I. Onwuezobe and F. Orok, "Associated risk factors of asymptomatic urinary tract infection among pregnant women attending antenatal care in a secondary health care facility in a southsouth Nigerian City," International Journal of Current Microbiology and Applied Sciences, vol. 8, no. 8, pp. 223-230, 2015.

[57] S.-E. Lee, K.-T. Kim, Y.-S. Park, and Y.-B. Kim, "Association between asymptomatic urinary tract infection and postoperative spine infection in elderly women: a retrospective analysis study," Journal of Korean Neurosurgical Society, vol. 47, no. 4, pp. 265-270, 2010.

[58] M. Vardi, T. Kochavi, Y. Denekamp, and H. Bitterman, "Risk factors for urinary tract infection caused by Enterobacteriaceae with extended spectrum beta-lactamase resistance in patients admitted to internal medicine departments," Sat, vol. 7, p. 20, 2012.

[59] P. Akerele, F. Abhulimen, and J. Okonofua, "Prevalence of asymptomatic bacteriuria among pregnant women in Benin City, Nigeria," Journal of Obstetrics and Gynaecology, vol. 21, no. 2, pp. 141-144, 2001. 